\title{
Imaging patterns and outcome of posterior reversible encephalopathy syndrome during childhood cancer treatment
}

\author{
Raja B. Khan, MD¹, Zsila S. Sadighi, MD ${ }^{1}$, Jennifer Zabrowski, RN¹, Amar Gajjar, MD², and \\ Sima Jeha, MD $^{3}$ \\ ${ }^{1}$ Division of Neurology, St. Jude Children's Research Hospital, Memphis, TN, USA \\ 2Department of Pediatric Medicine, St. Jude Children's Research Hospital, Memphis, TN, USA \\ ${ }^{3}$ Department of Oncology, St. Jude Children's Research Hospital, Memphis, TN, USA
}

\begin{abstract}
Background-Diagnosis of posterior reversible encephalopathy syndrome (PRES) requires presence of headache, seizures, impaired vision, or altered mentation accompanied by specific imaging findings. We aimed to study long-term clinical and radiologic outcome of PRES in children with cancer to augment limited available data.
\end{abstract}

Procedure-Retrospective review of children with cancer who were diagnosed with PRES.

Results-We identified PRES in 21 males and 16 females amongst the 5,217 children treated during the study period. Median time from cancer diagnosis to PRES was 6.6 months in 25 leukemia (1.6\%), 5 brain tumor $(0.3 \%)$, and 7 other solid tumor $(0.4 \%)$ patients; $\mathrm{p}=<0.0001$ for leukemia versus all other tumors. Symptoms included seizures (97\%), headaches (40\%), altered mentation (68\%), and vision impairment (27\%). Hypertension in $97 \%$ and steroids use was seen in $78 \%$. Headaches, visual disturbance, and mental status resolved within a median of $<3$ days, while epilepsy developed in 19\%. T2 hyperintense signal was present in $100 \%$ of occipital, $47 \%$ of temporal, $75 \%$ of parietal, and $55 \%$ of frontal lobes, as well as $22 \%$ of cerebellum and $5 \%$ of basal ganglia. Follow-up MRI in 34 patients showed partial or complete T2 resolution in 79\%, development of laminar necrosis in 5, microhemorrhages in 6 , and focal atrophy in 3 .

Conclusion-PRES in children is more common in hematological malignancy compared to other tumors and is associated with hypertension and steroid use. Seizure is the most common acute manifestation. Most MRI changes resolve, but persistent imaging abnormality and epilepsy may develop in a significant minority.

\section{Keywords}

PRES; childhood; cancer; outcome

"Correspondence to: Raja B. Khan, MD, 262 Danny Thomas Place, MS 135, Memphis, TN 38105, USA, Tel: 901-595-8575, Fax: 901-595-8548, raja.khan@stjude.org.

Financial disclosure: All authors have no financial relationships to disclose relevant to this article.

Conflict of Interest: There are no financial disclosures, conflicts of interest, and/or acknowledgements for the authors in regards to this manuscript.

Informed consent: Because of retrospective nature and minimal risk, need for informed consent was waived by the institutional review board. 


\section{INTRODUCTION}

Posterior reversible encephalopathy syndrome (PRES) is a clinico-radiological diagnosis characterized by a spectrum of symptoms, including altered mental status, headache, seizures and visual changes. Magnetic resonance imaging (MRI) evidence of hyperintense signal change in the cortical-subcortical areas, typically in posterior circulation distribution, is required to confirm PRES diagnosis [1, 2]. Initially described in hypertensive encephalopathy and eclampsia, subsequent reports have suggested an association with drugs, particularly immune system and vascular endothelial system modulating agents [3-5]. Outcome of PRES in non-cancer patients is usually good, but permanent sequelae and life threatening complications have been described [6,7]. Reports on outcomes of PRES in children with cancer have been limited by small number of patients and inadequate followup data $[8,9]$. We analyzed our single institution experience with PRES in a larger cohort than previously reported in order to better understand radiologic patterns and long-term outcome in respect to clinical features and radiologic resolution.

\section{METHODS}

We searched institutional databases between January 1994 and January 2014 for diagnosis of cerebral edema, posterior reversible encephalopathy syndrome, PRES, brain edema, and encephalopathy in children diagnosed with cancer at less than 18 years of age. Records were reviewed after obtaining Institutional Review Board permission. Diagnostic criteria included acute presentation with mental status change, visual impairment, headache, or seizure with concurrent T2 or Fluid Attenuated Inversion Recovery (FLAIR) hyperintensity in subcortical areas of the brain, or in brain stem and cerebellum. All MRI scans of suspected PRES in patients with childhood cancer were available and were reviewed by a single investigator (RBK). Demographic and pre specified clinical variables were collected on all patients.

Only 2 patients developed PRES before 2000 and were excluded from incidence calculation. We used the 5,217 total number of patients treated at our institution between January 2000 to January 2014 to calculate an approximate incidence rate of PRES. Two tailed Fisher Exact test was applied to compare incidence rates of PRES in different tumor populations.

\section{RESULTS}

We identified PRES in 37 patients (21 males and 16 females) treated between January 1994 and January 2014. Most common cancer was leukemia: 16 acute lymphoblastic; 5 acute myeloid; and 4 myelodysplastic. During this period a total of 5,217 children with leukemia $(n=1414)$, brain tumor $(n=1,872)$, and other solid tumor $(n=1,931)$ were treated at our institution. Thus, PRES was seen in $1.6 \%$ of children with leukemia, $0.3 \%$ of brain tumor, and $0.4 \%$ of other solid tumors. Difference in incidence rate was highly significant for leukemia versus brain tumors ( $\mathrm{p}=<0.0001)$, leukemia versus other solid tumors $(\mathrm{p}=0.0001)$, leukemia versus all other tumors $(\mathrm{p}=<0.0001$ ), but not for brain tumors versus other solid tumors $(\mathrm{p}=0.8)$. Clinical and demographic features are provided in Table 1 . Median age at 
cancer diagnosis was 9 years (range 1 to 17.5 years) and median time from cancer diagnosis to PRES was 6.6 months (range 0 to 101.4 months). Fifteen of 37 developed PRES while being treated for relapsed cancer and 15 had bone marrow transplant. Other tumors included 5 malignant brain tumors and 7 miscellaneous solid tumors. Twenty one (57\%) had received chemotherapy within 2 weeks of developing PRES (Table 2) and 12 had bone marrow transplant at a median time of 3.3 months (range 0.9 to 30.2 months) prior to PRES; 5 of the 12 with bone marrow transplant had chemotherapy within 2 weeks for relapsed disease.

\section{Clinical Presentation}

Twenty one (57\%) participants of this study were alive at a median follow-up of 27.5 months (range 0.6 to 214.5 months). Seizures were the most common clinical finding at PRES onset. Sixteen (43\%) patients had a single seizure, 5 (14\%) had 2, and $15(40 \%)$ had 3 or more seizures at PRES onset. Seizure types included simple or complex partial in 28 (7 with secondary generalization and 5 with status epilepticus), generalized tonic clonic in one, and seizure type could not be determined in one. Seizures came under control in all patients after initiation of anti-seizure medications. Initial anti-seizure therapy included intravenous benzodiazepine in 26 patients, phenytoin in 14, and levetiracetam in 22 . Three patients required addition of a second anti-seizure drug in the acute phase. Breakthrough seizures during follow-up developed in 9 while still receiving anti-seizure treatment and were controlled with dose adjustment of anti-seizure drugs. Maintenance anti-seizure medications included phenytoin in 3, phenobarbital in 1, gabapentin in 12, and levetiracetam in 22; one patient each required an addition of topiramate, lamotrigine, phenytoin, and valproic acid to control the seizures. More than 6 months seizure free status at last follow-up was present in $35(95 \%)$ and 2 had infrequent seizures $(<1 /$ month). Seven patients were still taking antiseizure medications and 5 of these had failed an attempted seizure medication withdrawal.

Visual changes at presentation were present in $27 \%$ of patients and resolved at a median of 2 days from onset (range $0-12$ days). Altered mental status was present in $68 \%$ returning to base line within a median of 2 days from onset (range 0-16 days). Headache was present in $40 \%$ and resolved in all within a median of 2.5 days (range $0-31$ days). Chronic headaches were not reported during follow-up visits.

\section{Laboratory findings}

Serum electrolytes and CBC were available in all patients at the onset of PRES symptoms and showed mild to moderate renal impairment (creatinine 2 to 5) in 3 patients, 2 had hypomagnesaemia ( $0.8 \mathrm{MEq} / \mathrm{L})$, and none had abnormal sodium levels, hypocalcaemia or thrombocytopenia. Modest elevations (2-3 fold) in liver enzymes were present in 7 and moderate elevation (4-5 fold) in 4 patients. There was no evidence of tumor lysis syndrome in any patient.

\section{Electroencephalogram (EEG)}

An EEG was obtained in the acute phase of illness after resolution of clinical seizure in 23 patients and showed focal slow waves in 14 and diffuse slow waves in 6. Epileptiform focal discharges were present in 10 and were bilateral in two patients. A follow-up EEG was obtained in 23 patients at a median time of 141 days after PRES (range 6 to 616 days) and 
showed focal slow waves in 9, and diffuse slow waves, focal and bilateral epileptiform discharges in one each.

\section{Neuroimaging findings}

MRI data is provided in Table 3 and Figures 1 and 2. First MRI was obtained within 96 hours in 36 patients and majority (89\%) of MRI were completed within 24 hours from presentation. Only a CT scan was ordered in one patient. Typical T2 hyperintense signal abnormalities were seen in the occipital lobes in all 36 MRI, with additional parietal, frontal, and temporal lobe white matter involvement in most. Central type of PRES with predominant basal ganglia involvement was seen in 2 patients. A repeat MRI was obtained in 34 patients at a median time of 29 days (range 6 to 711 days) and showed resolution of occipital white matter changes in $23(64 \%)$ and partial resolution in additional 4 subjects. Laminar necrosis had developed in $5(14 \%)$, microhemorrhages in $6(16 \%)$, and focal atrophy in $3(8 \%)$ of subjects. A third MRI was obtained in 22 subjects at a median time of 20.5 months (range 3.1 to 126.3) and showed persistent white matter changes in 4.

\section{DISCUSSION}

This report evaluates a large cohort of patients with an uncommon complication (PRES) of an uncommon childhood illness (cancer). Although our study is retrospective in nature, all patients were seen by a board certified neurologist with careful documentation of clinical events. Finally, good clinical and radiological follow-up data was available on almost all of the study subjects. We found seizure to be the most common (97\%) presenting symptom along with mental status change (68\%). This is consistent with earlier reports suggesting seizure being relatively more common presenting symptom of PRES in children compared to in adults [1]. Our results also suggest that a significant minority (19\%) will develop epilepsy and may require a long-term treatment with anti-seizure drugs. Reassuringly, seizures responded to anti-seizure therapy in all. However, 4 (11\%) did require an addition of a second anti-seizure drug. We want to emphasize that currently we do not use hepatic enzyme inducing anti-seizure drugs during cancer treatment. The use of such drugs in some patients of this cohort was at a time period when increased clearance of certain chemotherapeutic agents by enzyme inducing drugs was not recognized.

Another interesting feature of our cohort is that frontal lobe T2 hyperintense signal change in anterior circulation territory was as common as that in the parietal and temporal lobes. Earlier studies of predominantly children with PRES without concurrent cancer also reported similar findings $[1,6,10]$. In more severe cases we demonstrate presence of microhemorrhages suggesting that underlying pathophysiology may involve relaxation of tight endothelial junctions leading to edema and extravasation of red blood cells. None of the children had thrombocytopenia severe enough to explain these micro-hemorrhages.

Development of focal atrophy, laminar necrosis (Figure 2), or epilepsy in some patients with PRES suggests that a small subset of these patients is at risk of developing chronic sequelae. However, only one patient who developed epilepsy had focal atrophy and one had laminar necrosis suggesting no clinico-radiological association between these chronic sequelae. We also identified reversible vasospasm in large intracranial arteries in 3 patients. Magnetic 
resonance imaging may inaccurately assess the degree of vessel narrowing and investigation with CT angiogram or digital subtraction angiography will likely show a higher incidence of vessel caliber irregularity. Indeed, one adult study of 47 patients did show vessel caliber abnormalities on catheter angiography or magnetic resonance angiography in majority of patients with PRES [11]. This is consistent with the hypothesis of altered vasomotor auto regulation being a major contributor to development of PRES.

The retrospective nature of the data prevented us from performing risk factor analysis. Certain facts can however be gleaned from our data: PRES is more common during treatment of hematological malignancy compared to other tumors; hypertension is almost universal; and steroid use is very frequent. Less clear is the role of neurotoxic chemotherapy drugs used in hematological cancer, or release of cytokines during tumor kill. Several studies have cited hypertension, chemotherapy drugs, steroid use, tumor lysis syndrome, and induction phase of leukemia treatment as contributing to risk for PRES [2, 9, 12, 13]. However, there was no biochemical evidence of tumor lysis syndrome, or abnormal serum electrolytes present in majority of our cohort.

Aim of our study was to define long term clinical and radiologic outcomes of PRES in children with cancer. Although we succeeded in our aim, major limitations of our data are lack of neuro-cognitive assessment and absence of a control population to study the difference and risk factors. Risk factors can be analyzed in a more homogeneous population and should be prospectively studied. This may be possible in future protocols for treatment of acute lymphoblastic leukemia.

In conclusion, PRES is more common in children with hematological malignancies compared to other tumors, hypertension is present in majority of children at PRES onset, seizure and altered mental state are the most common presenting manifestations, and clinical and radiologic outcome is favorable in most with symptoms resolving in days. However, a small but significant minority will develop persistent brain imaging changes and epilepsy.

\section{ACKNOWLEDGEMENTS}

Funding: Supported by Cancer Center Support Grant CA21765 from the National Cancer Institute, and by the American Lebanese Syrian Associated Charities.

\section{Abbreviations}

PRES Posterior reversible encephalopathy syndrome

FLAIR Fluid attenuated inversion recovery

MRI Magnetic resonance imaging

EEG Electroencephalogram

TBI Total body irradiation

BMI Body mass index 


\section{REFERENCES}

1. Siebert E, Bohner G, Endres M, Liman TG. Clinical and radiological spectrum of posterior reversible encephalopathy syndrome: does age make a difference?-A retrospective comparison between adult and pediatric patients. PLoS One. 2014

2. Lee VH, Wijdicks EF, Manno EM, Rabinstein AA. Clinical spectrum of reversible posterior leukoencephalopathy syndrome. Arch Neurol. 2008; 65:205-210. [PubMed: 18268188]

3. Burnett MM, Hess CP, Roberts JP, Bass NM, Douglas VC, Josephson SA. Presentation of reversible posterior leukoencephalopathy syndrome in patients on calcineurin inhibitors. Clin Neurol Neurosurg. 2010; 112:886-891. [PubMed: 20800343]

4. Khurana A, Dasanu CA. Posterior reversible encephalopathy syndrome due to targeted agents: vemurafinib among suspects! J Oncol Pharm Pract. 2014 pii: 1078155214543212. PMID: 25013187.

5. Myint ZW, Sen JM, Watts NL, Druzgal TJ, Nathan BR, Ward MD, Boyer JE, Fracasso PM. Reversible posterior leukoencephalopathy syndrome during regorafenib treatment: a case report and literature review of reversible posterior leukoencephalopathy syndrome associated with multikinase inhibitors. Clin Colorectal Cancer. 2014; 13:127-130. [PubMed: 24461491]

6. Siebert E, Spors B, Bohner G, Endres M, Liman TG. Posterior reversible encephalopathy syndrome in children: radiological and clinical findings - a retrospective analysis of a German tertiary care center. Eur J Paediatr Neurol. 2013; 17:169-175. [PubMed: 22954513]

7. Cordelli DM, Masetti R, Ricci E, Toni F, Zama D, Maffei M, Gentili A, Parmeggiani A, Pession A, Franzoni E. Life-threatening complications of posterior reversible encephalopathy syndrome in children. Eur J Paediatr Neurol. 2014; 18:632-640. [PubMed: 24814477]

8. Kheir JN, Lawlor MW, Ahn ES, Lehmann L, Riviello JJ, Silvera VM, McManus M, Folkerth RD. Neuropathology of a fatal case of posterior reversible encephalopathy syndrome. Pediatr Dev Pathol. 2010; 13:397-403. [PubMed: 20158377]

9. Morris EB, Laningham FH, Sandlund JT, Khan RB. Posterior reversible encephalopathy syndrome in children with cancer. Pediatr Blood Cancer. 2007; 48:152-159. [PubMed: 16317748]

10. Donmez FY, Guleryuz P, Agildere M. MRI Findings in Childhood PRES: What is Different than the Adults? Clin Neuroradiol. 2014 PMID: 25293448.

11. Bartynski WS, Boardman JF. Catheter angiography, MR angiography, and MR perfusion in posterior reversible encephalopathy syndrome. AJNR Am J Neuroradiol. 2008; 29:447-455. [PubMed: 18079186]

12. Gümüş H, Per H, Kumandaş S, Yikilmaz A. Reversible posterior leukoencephalopathy syndrome in childhood: report of nine cases and review of the literature. Neurol Sci. 2010; 31:125-131. [PubMed: 19809787]

13. Suzuki D, Kobayashi R, Iguchi A, Sano H, Kishimoto K, Yasuda K, Kobayashi K. Tumor lysis syndrome as a risk factor for posterior reversible encephalopathy syndrome in children with hematological malignancies. Int J Hematol. 2014; 100:485-489. [PubMed: 25216697] 

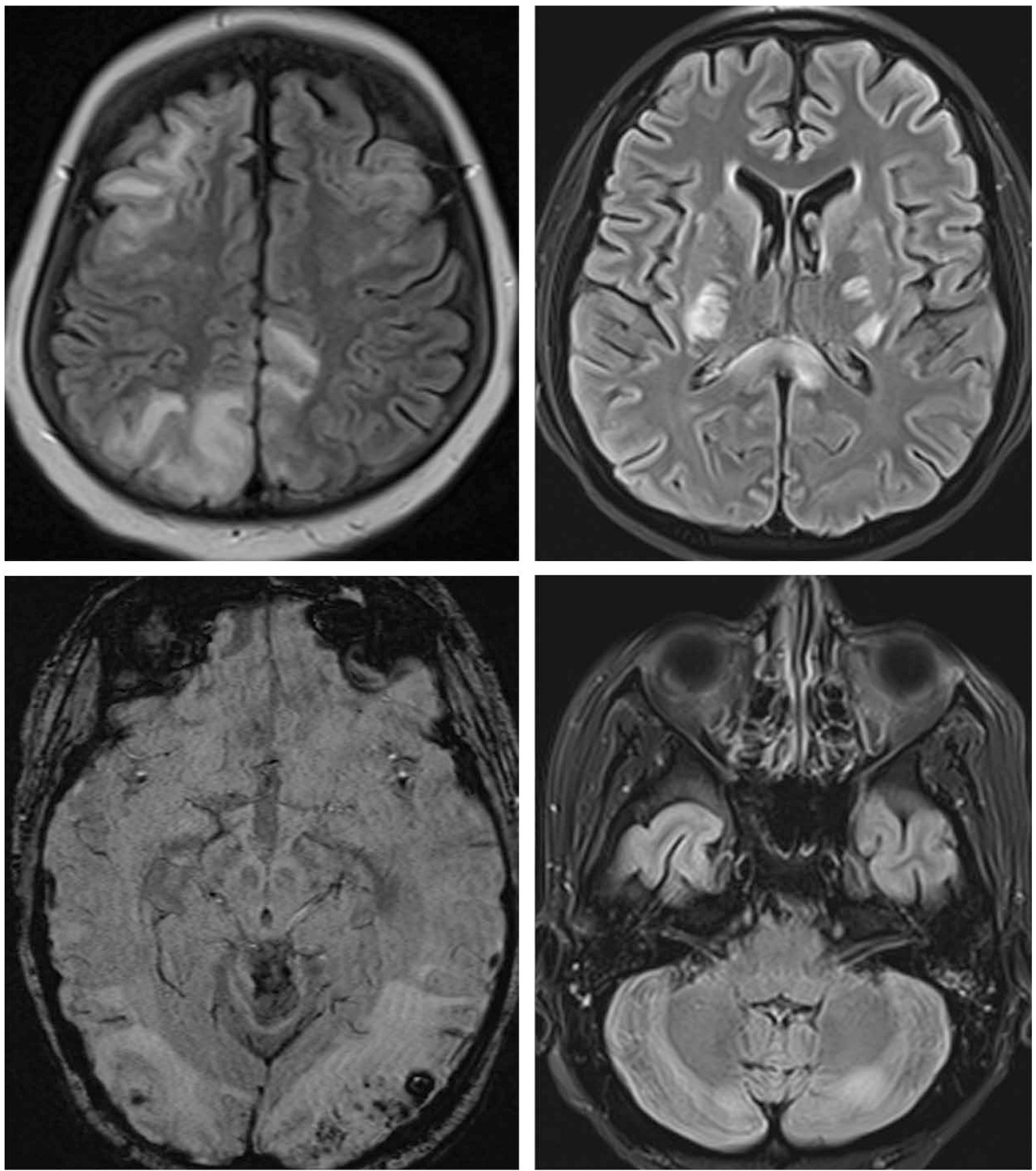

Figure 1.

FLAIR images showing edema involving white matter and cortex in occipital, parietal and frontal lobes in top left; central variant of PRES with edema in internal capsule, corpus callosum and basal ganglia in top right; and cerebellar grey and white matter in bottom right. Susceptibility weight image showing multiple micro hemorrhages in bilateral occipital area in the bottom left image. 

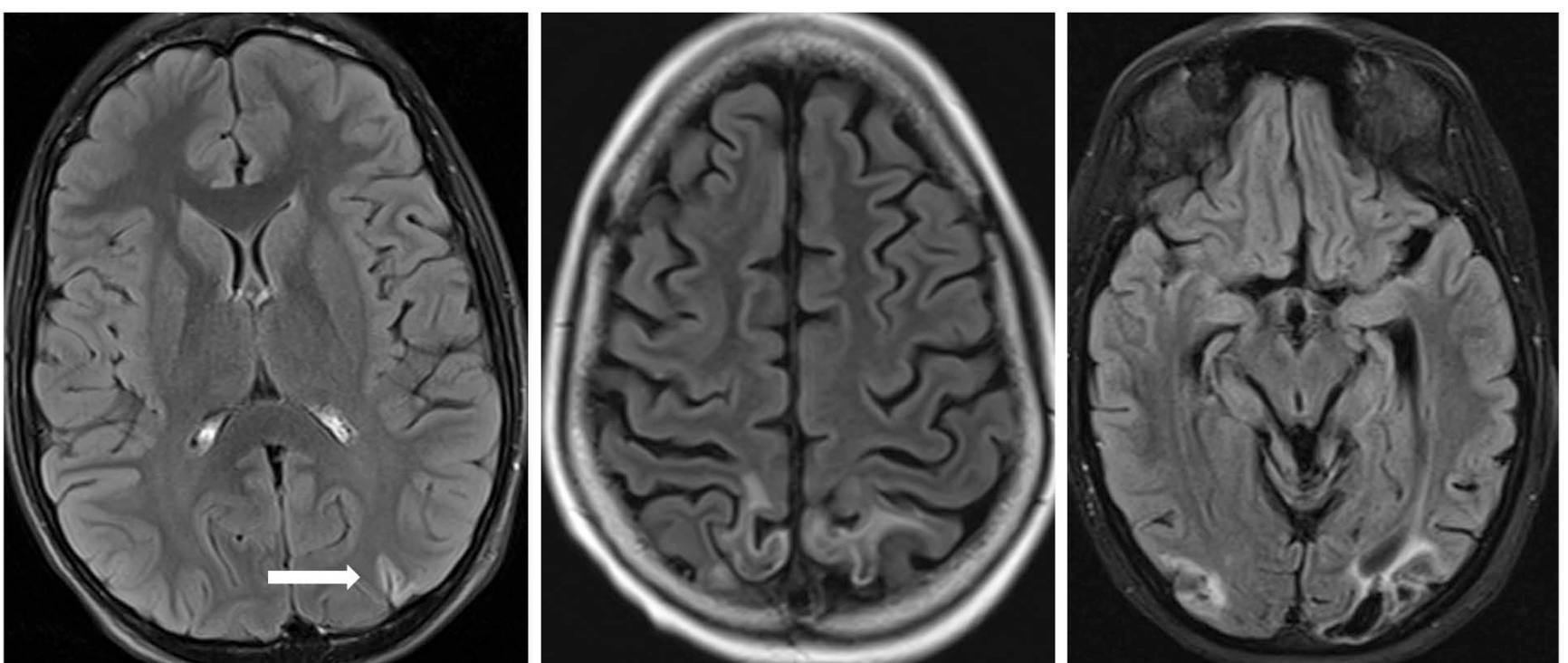

Figure 2.

Post contrast FLAIR images showing varying degree of laminar necrosis and focal atrophy in follow up scans. 
Table I

Clinical and demographic features of 37 children with cancer who developed PRES

\begin{tabular}{|c|c|}
\hline Gender Male: Female & $21: 16$ \\
\hline Median age at cancer diagnosis & 9 years (range 1 to 17.5 ) \\
\hline Median time to PRES & 6.6 months ( 0 to 101.4 ) \\
\hline \multicolumn{2}{|l|}{ Tumor type } \\
\hline Leukemia: & 25 \\
\hline Brain tumor & 5 \\
\hline Other solid tumor & 7 \\
\hline Relapsed Leukemia & 8 \\
\hline \multicolumn{2}{|l|}{ Hypertension: } \\
\hline Borderline & 1 \\
\hline Definite & 35 \\
\hline Chemotherapy within 14 days & 21 \\
\hline \multicolumn{2}{|l|}{ Radiation: } \\
\hline Total body & 9 \\
\hline Brain & 7 \\
\hline Calcineurin inhibitors & 9 \\
\hline Steroids & 29 \\
\hline \multicolumn{2}{|l|}{ BMI: } \\
\hline $20-25:$ & 5 \\
\hline$>25$ & 6 \\
\hline Headache & 15 \\
\hline Seizures & 36 \\
\hline Breakthrough seizures & 9 \\
\hline Vision impairment & 10 \\
\hline Altered mental status & 25 \\
\hline Recurrent PRES & 2 \\
\hline
\end{tabular}

PRES) posterior reversible encephalopathy syndrome, TBI) total body irradiation, BMI) body mass index 
Table II

Chemotherapy and immunosuppression within two weeks of PRES $(n=21)$

\begin{tabular}{|c|c|}
\hline Vincristine & 9 \\
\hline Etoposide & 7 \\
\hline $\begin{array}{l}\text { Cytarabine } \\
\text { Intrathecal } \\
\text { Intravenous }\end{array}$ & $\begin{array}{l}9 \\
2\end{array}$ \\
\hline $\begin{array}{c}\text { Methotrexate } \\
\text { Intrathecal } \\
\text { Intravenous }\end{array}$ & $\begin{array}{l}10 \\
3\end{array}$ \\
\hline $\begin{array}{l}\text { Asparaginase } \\
\text { Steroids }\end{array}$ & $\begin{array}{c}4 \\
29\end{array}$ \\
\hline Daunorubicin & 4 \\
\hline Cyclophosphamide & 3 \\
\hline Dasatinib & 1 \\
\hline Infliximab/rituximab & 2 \\
\hline Bevacizumab & 1 \\
\hline Calcineurin inhibitors & 9 \\
\hline
\end{tabular}

로을

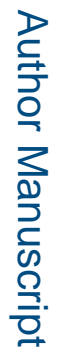

Pediatr Blood Cancer. Author manuscript; available in PMC 2017 March 01. 
Table III

MRI T2-hyperintense signal change involvement

\begin{tabular}{|c|c|c|c|}
\hline Area & Initial MRI (n=36) & $2^{\text {nd }} \operatorname{MRI}(n=34)$ & $3^{\text {rd }}$ MRI $(n=22)$ \\
\hline Frontal white matter & 20 & 1 & 0 \\
\hline Parietal white matter & 27 & 3 (1 improved) & 1 \\
\hline Temporal white matter & 17 & 2 (2 improved) & 1 \\
\hline Occipital white matter & 36 & 13 (4 improved) & 4 \\
\hline Cerebellum & 8 & 0 & 0 \\
\hline Basal ganglia & 2 & 0 & 0 \\
\hline Thalamus & 1 & 0 & 0 \\
\hline Brain stem & 0 & 0 & 0 \\
\hline Cortical gray & 36 & 12 (4 improved) & 3 \\
\hline Hemorrhage & 5 & 6 & 3 \\
\hline Laminar necrosis & 0 & 5 & 1 \\
\hline Focal atrophy & 0 & 3 & 3 \\
\hline Contrast enhancement & 4 & 1 (1 improved) & 0 \\
\hline MRA stenosis $(n=21)$ & 3 & 0 & 0 \\
\hline Diffusion restriction $(\mathrm{n}=33)$ & 8 & 0 & 0 \\
\hline
\end{tabular}

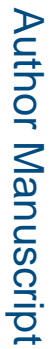

를

.

